\title{
On asymptotic continuity of functions of quantum states
}

\author{
Barbara Synak-Radtke ${ }^{(1)}$ and Michał Horodecki ${ }^{(1)}$ \\ ${ }^{(1)}$ Institute of Theoretical Physics and Astrophysics, University of Gdańsk, Poland
}

\begin{abstract}
A useful kind of continuity of quantum states functions in asymptotic regime is so-called asymptotic continuity. In this paper we provide general tools for checking if a function possesses this property. First we prove equivalence of asymptotic continuity with so-called robustness under admixture. This allows us to show that relative entropy distance from a convex set including maximally mixed state is asymptotically continuous. Subsequently, we consider arrowing - a way of building a new function out of a given one. The procedure originates from constructions of intrinsic information and entanglement of formation. We show that arrowing preserves asymptotic continuity for a class of functions (so-called subextensive ones). The result is illustrated by means of several examples.
\end{abstract}

\section{INTRODUCTION}

One of basic issues of Quantum Information Theory is to evaluate operational quantities such as capacities of quantum (usual of teleportation) channel [1, 2] costs creating quantum states under some natural constraints [3, 4], compression rates [5] or localisable information rates [6, 7]. The quantities are usually defined in spirit of Shannon - in asymptotic regime of many uses of channel or many copies of state. Apart from such operational quantities one also considers mathematical functions, that are expected to reflect somehow those features of states or channels. To this end, one chooses functions, that satisfy some requirements. For example, most of entanglement measures are mathematical functions, that do not increase under local operations and classical communication [3, 8]. Other examples are correlation measures (see e.g. 9, 10, 11, 12]). Such functions turn out to be very useful, as they often provide upper or lower bounds for operational quantities. in asymptotic regime, the functions are especially useful, if they are asymptotically continuous. The prototype for asymptotic continuity is Fannes inequality [13] for von Neumann entropy $S(\varrho)=-\operatorname{Tr} \varrho \log \varrho$. which says that for any states $\varrho$ and $\sigma$ with $\|\varrho-\sigma\|_{1} \leq 1 / 2$ we have

$$
|S(\varrho)-S(\rho)| \leq\|\varrho-\sigma\|_{1} \log d+\eta\left(\|\varrho-\sigma\|_{1}\right)
$$

where $\eta(x)=-x \log x, d$ is dimension of Hilbert space. The important feature of this stronger form of continuity, is that the right-hand-side scales logarithmically with dimension of Hilbert space. This kind of inequality, was first applied in quantum information theory in [14, 15] to provide lower bound for compression rates of mixed signal states (interestingly, the question of achievability of the bound is in general still open). Subsequently, it was applied to entanglement theory [16] which lead, in particular, to methods of providing bounds for distillable entanglement and entanglement cost [4, 17]. Asymptotic continuity has become an important tool in proving irreversibility of pure states transformations (see [18] and references therein).

In [19, 20] two measures of entanglement have been proven to satisfy Fannes-like inequality (i.e. to be asymptotically continuous) - entanglement of formation $E_{F}[\underline{3}$ and relative entropy of entanglement [21]. In 22] asymptotic continuity of conditional entropy $S(A \mid B)=S\left(\varrho_{A B}\right)-S\left(\varrho_{B}\right)$ have been proven, where the right-hand-side depend only on dimension of system $A$. This allowed to prove asymptotic continuity of third measure of entanglement - squashed entanglement [23]. The importance of asymptotic continuity was made even more transparent in 24] where it was shown, that a convex and so called subextensive function, if not asymptotically continuous, it behaves in a quite weird way: namely, after removing one qubit, it can change at arbitrarily large amount.

Clearly, it is very important to know whether a function is asymptotically continuous or not. Yet it is usually rather a difficult task. The aim of this paper is to provide general tools for checking asymptotic continuity. First, we show that the latter is equivalent to so called "robustness under admixtures", i.e. a function is asymptotically continuous, if it does not change too much under admixing any state with a small weight. Using it, we prove that relative entropy distance from any convex set including maximally mixed state is asymptotically continuous, extending therefore result of [20] where it was proven for compact and convex sets.

Next, we consider a procedure, called arrowing, of building new functions out of given functions. The procedure originates both from classical privacy theory $[25,26]$ - where the prototype was so-called intrinsic information - as well as from entanglement theory, since it includes as a special case the other procedure called convex roof [27], the prototype of which was entanglement of formation [3]. Since arrowing is commonly used in different contexts (see quite recent application [28]), it is important to be able to check the properties of arrowed versions of different functions. We provide here a quite general result, showing that for subextensive functions such procedure preserve asymptotic continuity, i.e. if an original function is asymptotically continuous, so is its "arrowed" version. We then apply it to show, that some tripartite entanglement measure [18, 29] as well as so called mixed convex roof of quantum mutual information introduced in [25] are asymptotically continuous. 


\section{BASIC DEFINITIONS}

In this section we will introduce some definitions which we will use throughout this paper. Set of states. A positive operator $\varrho \in \mathcal{S}$ with $\operatorname{Tr} \varrho=1$, acting on Hilbert space $\mathcal{H}$ we will call state. Set of all states will be denoted by $\mathcal{S}(\mathcal{H})$. (We will deal with finite dimensional Hilbert spaces). A state is called pure, if it is of the form $|\psi\rangle\langle\psi|$ where $\psi \in \mathcal{H}$. Otherwise it is called mixed state.

Von Neumann entropy $S(\varrho)$ for a state $\varrho$ is given by formula:

$$
S(\varrho)=-\operatorname{Tr} \varrho \log \varrho
$$

We use base 2 logarithm in this paper.

Relative entropy for states $\varrho$ and $\sigma$ is defined as:

$$
S(\varrho \mid \sigma)=\operatorname{Tr} \varrho \log \varrho-\operatorname{Tr} \varrho \log \sigma
$$

Trace norm of an operator A is given by:

$$
\|A\|_{1}=\operatorname{Tr} \sqrt{A A^{\dagger}}
$$

where $A^{\dagger}$ stands for Hermitian conjugation.

Measurement. We will consider measurements with finite number of outcomes, represented by finite sets of operators $\mathcal{M}=\left\{A_{i}\right\}$ satisfying $\sum_{i} A_{i}^{\dagger} A_{i}=I$. Slightly abusing terminology, we will call the measurements POVMs (Positive Operator Valued Measure).

Subextensivity A function $f: \mathcal{S}(\mathcal{H}) \rightarrow R$ is subextensive if

$$
\forall_{\varrho} \quad \exists_{M} \quad f(\varrho) \leq M \log d
$$

where $M$ is constant, $d=\operatorname{dim} \mathcal{H}$.

Definition 1 Let $f$ be a real-valued function $f: S\left(\mathcal{C}^{d}\right) \mapsto \mathcal{R}$ and $\varrho_{1}, \varrho_{2}$ are states acting on Hilbert space $\mathcal{C}^{d}$ and $\varepsilon=\left\|\varrho_{1}-\varrho_{2}\right\|_{1}$. Then a function is asymptotically continuous if fulfills the following condition

$$
\forall_{\varrho_{1}, \varrho_{2}}\left|f\left(\varrho_{1}\right)-f\left(\varrho_{2}\right)\right| \leq K_{1} \varepsilon \log d+O(\varepsilon)
$$

where $K_{1}$ is constant and $O(\varepsilon)$ is any function, which satisfies the condition that $O(\varepsilon)$ converges to 0 when $\varepsilon$ converges to 0 and depends only on $\varepsilon$. (In particular, it does not depend on dimension).

Definition 2 Let $f$ be a real-valued function $f: S\left(\mathcal{C}^{d}\right) \mapsto \mathcal{R}$ and $\varrho_{1}, \varrho_{2}$ are states acting on Hilbert space $\mathcal{C}^{d}$. Then a function is robust under admixtures if

$$
\forall_{\varrho_{1}, \varrho_{2}} \forall_{\delta>0}\left|f\left((1-\delta) \varrho_{1}+\delta \varrho_{2}\right)-f\left(\varrho_{1}\right)\right| \leq K_{2} \delta \log d+O(\delta)
$$

where $K_{2}$ is constant and $O(\delta)$ is any function, which satisfies the condition that $O(\delta)$ converges to 0 when $\delta$ converges to 0 and depends only on $\delta$. (In particular, it does not depend on dimension).

Remark. Notice that usually for asymptotic continuity or robustness under admixtures we will not require fulfilling conditions (6) and (7) for whole range of $\varepsilon$ or $\delta$. We will rather restrict to some limited subset of positive real value of $\varepsilon$ or $\delta$ (limited by 1 or $\frac{1}{2}$, for example.)

\section{ASYMPTOTIC CONTINUITY AND ROBUSTNESS UNDER SMALL ADMIXTURES.}

In this section we prove equivalence between asymptotic continuity and robustness under admixtures of function. This is an extension of result of [24], where it is proved that if a function $f$, under admixtures does not change more than a constant, and subextensive then is also asymptotically continuous.

Proposition 1 Let $f$ be a function $f: S\left(\mathcal{C}^{d}\right) \mapsto \mathcal{R}$ then the function is asymptotically continuous if only if is robust under admixtures. 
Remark. This proposition can be also proved when we do not require "Lipschitz type" continuities, but rather "Cauchy type" ones. (See appendix)

\section{Proof.}

$" \Rightarrow "$

We assume that function is asymptotically continuous. This implies

$$
\begin{aligned}
& \left|f\left((1-\delta) \varrho_{1}+\delta \varrho_{2}\right)-f\left(\varrho_{1}\right)\right| \leq K_{1}\left\|\varrho_{1}-\left((1-\delta) \varrho_{1}+\delta \varrho_{2}\right)\right\|_{1} \log d+O\left(\left\|\varrho_{1}-\left((1-\delta) \varrho_{1}+\delta \varrho_{2}\right)\right\|_{1}\right)= \\
& =K_{1}|| \delta \varrho_{1}-\delta \varrho_{2} \|_{1} \log d+O\left(\left\|\delta \varrho_{1}-\delta \varrho_{2}\right\|_{1}\right) \leq 2 K_{1} \delta \log d+O(2 \delta)
\end{aligned}
$$

Lets take $K_{2}=2 K_{1}$. Then

$$
\left|f\left((1-\delta) \varrho_{1}+\delta \varrho_{2}\right)-f\left(\varrho_{1}\right)\right| \leq K_{2} \delta \log d+O(\delta)
$$

$" \Rightarrow "$

We will base on result of Refs. 22] (see also [30]), which can be viewed as a sort of generalized Tales theorem

$$
\forall_{\varrho_{1}, \varrho_{2}} \quad \exists_{\sigma, \gamma_{1} \gamma_{2}} \quad \sigma=(1-\varepsilon) \varrho_{1}+\varepsilon \gamma_{1}=(1-\varepsilon) \varrho_{2}+\varepsilon \gamma_{2}
$$

where $\varrho_{1}, \varrho_{2}, \sigma, \gamma_{1}, \gamma_{2}$ are states acting on Hilbert space and $\varepsilon=\left\|\varrho_{1}-\varrho_{2}\right\|_{1}$. Using it we obtain:

$$
\begin{aligned}
& \left|f\left(\varrho_{2}\right)-f\left(\varrho_{1}\right)\right| \leq\left|f\left(\varrho_{2}\right)-f(\sigma)\right|+\left|f(\sigma)-f\left(\varrho_{1}\right)\right|= \\
& \left|f\left((1-\varepsilon) \varrho_{2}+\varepsilon \gamma_{2}\right)-f\left(\varrho_{2}\right)\right|+\left|f\left((1-\varepsilon) \varrho_{1}+\varepsilon \gamma_{1}\right)-f\left(\varrho_{1}\right)\right| \leq 2 K_{2} \varepsilon \log d+2 O(\varepsilon)
\end{aligned}
$$

so that we can take $K_{1}=2 K_{2}$. Then

$$
\left|f\left(\varrho_{2}\right)-f\left(\varrho_{1}\right)\right| \leq K_{1} \varepsilon \log d+O(\varepsilon)
$$

This ends the proof.

\section{A. Application: asymptotic continuity of relative entropy distance from convex set of states.}

In 20] it was shown that so called relative entropy distance from convex, compact set including maximally state $\frac{I}{d}$ is asymptotically continuous. The proof was quit involved. Here, basing on Proposition 1 we present a more general result, where we do not require compactness of the set. Moreover our proof is straighter.

Relative entropy of distance $E_{R}^{\mathcal{D}}$ is defined as follows

$$
E_{R}^{\mathcal{D}}(\varrho)=\inf _{\sigma \in \mathcal{D}} S(\varrho \mid \sigma)
$$

where $\mathcal{D}$ is a convex set of state including maximally mixed state, $\varrho \in \mathcal{C}^{d}$.

We start with lemma:

Lemma 1 Relative entropy of distance $E_{R}^{\mathcal{D}}$ fulfills the following condition

$$
\left|E_{R}^{\mathcal{D}}((1-\varepsilon) \varrho+\varepsilon \sigma)-E_{R}^{\mathcal{D}}(\varrho)\right| \leq 2 \varepsilon \log d+H(\varepsilon)
$$

where $H(\varepsilon)=-\varepsilon \log \varepsilon-(1-\varepsilon) \log (1-\varepsilon)$

Proof.

First we show the that $E_{R}^{\mathcal{D}}$ satisfies the following inequality

$$
\sum_{k} p_{k} E_{R}^{\mathcal{D}}\left(\varrho_{k}\right)-E_{R}^{\mathcal{D}}\left(\sum_{k} p_{k} \varrho_{k}\right) \leq S\left(\sum_{k} p_{k} \varrho_{k}\right)-\sum_{k} p_{k} S\left(\varrho_{k}\right)
$$

This fact was shown for relative entropy distance from separable states in [31], but it is also true for relative entropy distance from any convex set of states. Here we repeat this proof for $E_{R}^{\mathcal{D}}$ defined in (15). Notice that for $\varrho=\sum_{k} p_{k} \varrho_{k}$

$$
\begin{aligned}
& S(\varrho \mid \sigma)=S\left(\sum_{k} p_{k} \varrho_{k} \mid \sigma\right)=\operatorname{Tr}\left(\sum_{k} p_{k} \varrho_{k} \log \left(\sum_{k} p_{k} \varrho_{k}\right)-\sum_{k} p_{k} \varrho_{k} \log \sigma\right)= \\
& =\operatorname{Tr}\left(\sum_{k} p_{k}\left(\varrho_{k} \log \varrho_{k}-\varrho_{k} \log \sigma+\varrho_{k} \log \varrho-\varrho_{k} \log \varrho_{k}\right)\right)= \\
& =\sum_{k} p_{k} S\left(\varrho_{k} \mid \sigma\right)+\sum_{k} p_{k} S\left(\varrho_{k}\right)-S(\varrho)
\end{aligned}
$$


Let $\sigma \in \mathcal{D}$ be a state such that $E_{R}^{\mathcal{D}}=S(\varrho \mid \sigma)-\delta$. Then we can rewrite

$$
E_{R}(\varrho)=\sum_{k} p_{k} S\left(\varrho_{k} \mid \sigma\right)+\sum_{k} p_{k} S\left(\varrho_{k}\right)-S(\varrho)-\delta \geq \sum_{k} p_{k} E_{R}\left(\varrho_{k}\right)+\sum_{k} p_{k} S\left(\varrho_{k}\right)-S(\varrho)-\delta
$$

Since by definition of $E_{R}^{\mathcal{D}} \delta$ can be arbitrarily small, we obtain

$$
\sum_{k} p_{k} E_{R}\left(\varrho_{k}\right)-E_{R}\left(\sum_{k} p_{k} \varrho_{k}\right) \leq S\left(\sum_{k} p_{k} \varrho_{k}\right)-\sum_{k} p_{k} S\left(\varrho_{k}\right)
$$

We use also fact that 32

$$
S\left(\sum_{k} p_{k} \varrho_{k}\right) \leq \sum_{k} p_{k} S\left(\varrho_{k}\right)+H\left(\left\{p_{k}\right\}\right)
$$

and that relative entropy distance is convex function, what is implied by convexity of quantum relative entropy in two arguments. Notice also that $E_{R}$ is bounded by $\log d$, because $\mathcal{D}$ includes maximally mixed state $\left(\right.$ so $E_{R} \leq S\left(\varrho \mid \frac{I}{d}\right)=$ $\log d-S(\varrho) \leq \log d)$. Then we have

$$
\begin{aligned}
& \left|E_{R}((1-\varepsilon) \varrho+\varepsilon \sigma)-E_{R}(\varrho)\right|=\left|E_{R}((1-\varepsilon) \varrho+\varepsilon \sigma)-(1-\varepsilon) E_{R}(\varrho)-\varepsilon E_{R}(\sigma)-\varepsilon E_{R}(\varrho)+\varepsilon E_{R}(\sigma)\right| \leq \\
& =\left|E_{R}((1-\varepsilon) \varrho+\varepsilon \sigma)-(1-\varepsilon) E_{R}(\varrho)-\varepsilon E_{R}(\sigma)\right|+\varepsilon\left|E_{R}(\varrho)\right|+\varepsilon\left|E_{R}(\sigma)\right| \\
& =(1-\varepsilon) E_{R}(\varrho)+\varepsilon E_{R}(\sigma)-E_{R}((1-\varepsilon) \varrho+\varepsilon \sigma)+\varepsilon\left|E_{R}(\varrho)\right|+\varepsilon\left|E_{R}(\sigma)\right| \\
& \leq S((1-\varepsilon) \varrho+\varepsilon \sigma)-(1-\varepsilon) S(\varrho)-\varepsilon S(\sigma)+\varepsilon \log d+\varepsilon \log d \leq H(\varepsilon)+2 \varepsilon \log d
\end{aligned}
$$

This ends the proof.

Remark. Note that the main feature of $E_{R}^{\mathcal{D}}$ responsible for robustness under admixtures, are the following: 1) $E_{R}^{\mathcal{D}}$ satisfy inequality :

$$
\left|E_{R}\left(\sum_{k} p_{k} \varrho_{k}\right)-\sum_{k} p_{k} E_{R}\left(\varrho_{k}\right)\right| \leq H\left(\left\{p_{k}\right\}\right)
$$

2) $E_{R}^{\mathcal{D}}$ is bounded by $\log d$.

Lemma 2 Relative entropy of distance $E_{R}$ is asymptotic continuous i.e.

$$
\left|E_{R}(\varrho)-E_{R}(\sigma)\right| \leq 4 \varepsilon \log d+2 H(\varepsilon)
$$

where $H(\varepsilon)=-\varepsilon \log \varepsilon-(1-\varepsilon) \log (1-\varepsilon)$ and $\varepsilon=\|\varrho-\sigma\|_{1}$.

Proof.

$E_{R}$ is robust under admixtures so under Proposition 1 is also asymptotically continuous.

\section{ASYMPTOTIC CONTINUITY OF FUNCTIONS BUILT BY "ARROWING"}

In this section we consider "arrowing" - a construction that from given function $f$ creates a new function denoted by $f_{\downarrow}$. The definition is motivated by intrinsic information and its generalizations [33, 34, 35]. The new function $f_{\downarrow}$ is defined on enlarged system as follows

Definition 3 For any function $f: \mathcal{S}\left(\mathcal{H}_{X}\right) \rightarrow R$ acting on states of system $X$, we define function $f_{\downarrow}: \mathcal{S}\left(\mathcal{H}_{X} \otimes \mathcal{H}_{E}\right) \rightarrow R$ as follows

$$
f_{\downarrow}\left(\rho_{X E}\right)=\inf _{\left\{A_{i}\right\}} \sum_{i} p_{i} f\left(\rho_{X}^{i}\right)
$$

where infimum is taken over all finite POVM's $\left\{A_{i}\right\}$ performed on system $E$ and

$$
p_{i}=\operatorname{Tr}\left(I_{X} \otimes A_{i}\right) \rho_{X E}, \quad \rho_{X}^{i}=\frac{1}{p_{i}} \operatorname{Tr}_{E}\left(I_{X} \otimes A_{i} \rho_{X E} I_{X} \otimes A_{i}^{\dagger}\right)
$$

i.e. $p_{i}$ is probability of outcome $i$, and $\rho_{X}^{i}$ is the state of system $X$ given outcome $i$ was obtained. 
Remark. We can define modified version of previous function as follows:

Definition 4 For any function $f: \mathcal{S}\left(\mathcal{H}_{X}\right) \rightarrow R$ acting on states of system $X$, we define function $f_{\uparrow}: \mathcal{S}\left(\mathcal{H}_{X} \otimes \mathcal{H}_{E}\right) \rightarrow R$ as follows

$$
f_{\uparrow}\left(\rho_{X E}\right)=\sup _{\left\{A_{i}\right\}} \sum_{i} p_{i} f\left(\rho_{X}^{i}\right)
$$

where supremum is taken over all finite POVM's $\left\{A_{i}\right\}$ performed on system $E$ and

$$
p_{i}=\operatorname{Tr}\left(I_{X} \otimes A_{i}\right) \rho_{X E}, \quad \rho_{X}^{i}=\frac{1}{p_{i}} \operatorname{Tr}_{E}\left(I_{X} \otimes A_{i} \rho_{X E} I_{X} \otimes A_{i}^{\dagger}\right)
$$

i.e. $p_{i}$ is probability of outcome $i$, and $\rho_{X}^{i}$ is the state of system $X$ given outcome $i$ was obtained.

All features of $f_{\downarrow}$ presenting in this paper are also valid for function $f_{\uparrow}$.

We have the following lemma, which is proven in Sec. IX

Lemma 3 The infimum in the definition of $f_{\downarrow}$ is achievable.

We will show in this section, that that asymptotic continuity and subextensivity of function $f$ implies asymptotic continuity of $f_{\downarrow}$. Thus in a sense, arrowing preserves asymptotic continuity. Let us stress that all the involved systems are finite-dimensional.

We will need the following definition:

Definition 5 Given a function $f$ defined on states of a system $X$, we define its conditional version $F$ for a quantumclassical state of a system $X E$

$$
\rho_{X E}^{q c}=\sum_{i} p_{i} \rho_{X}^{i} \otimes|i\rangle_{E}\langle i|
$$

as follows

$$
F\left(\rho_{X E}^{q c}\right)=\sum_{i} p_{i} f\left(\rho_{X}^{i}\right)
$$

If the quantum classical state was obtained from state $\rho_{X E}$ by a POVM $\mathcal{M}$ performed on system E we will also use notation $F\left(\rho_{X E}, \mathcal{M}\right) \equiv F\left(\rho_{X E}^{q c}\right)$.

Let us now present the main result of this section.

Proposition 2 Let $f$ be a function defined on states of system $X$, which is subextensive and asymptotic continuous. Then function $f_{\downarrow}$ is also asymptotically continuous. Moreover, the constant in asymptotic continuity condition depends only on dimension of system $X$.

\section{Proof.}

Let $\varrho_{X E}$ and $\sigma_{X E}$ be states and $\varepsilon=\left\|\varrho_{X E}-\sigma_{X E}\right\|_{1}$. Let $\mathcal{M}_{\varrho}=\left\{A_{k}^{\varrho}\right\}$ and $\mathcal{M}_{\sigma}=\left\{A_{k}^{\sigma}\right\}$ be the optimal measurements for $\rho$ and $\sigma$ respectively (i.e. the ones achieving infimum in definition of $f_{\downarrow}$ ) where $\sum_{k} A_{k}^{\varrho \dagger} A_{k}^{\varrho}=I_{E}, \sum_{k} A_{k}^{\sigma \dagger} A_{k}^{\sigma}=I_{E}$. For measurement $\mathcal{M}_{\sigma}$, let $p_{k}$ and $q_{k}$ be probabilities of outcomes if a state was $\varrho$ and $\sigma$ respectively. The resulting states on system $X$, given the outcome was $k$, we will denote by $\varrho_{k}$ and $\sigma_{k}$ respectively. Due to asymptotic continuity (see sec. III) we assume that

$$
\left|f\left(\varrho_{X E}\right)-f\left(\sigma_{X E}\right)\right| \leq K \varepsilon \log d_{X}+O(\varepsilon)
$$

and due to subextensivity

$$
|f(\rho)| \leq M \log d_{X}
$$


for any state $\rho$ on system $X$, where $d_{X}=\operatorname{dim} \mathcal{H}_{X}$ and $M$ and $K$ are constants. Then we have the following estimate

$$
\begin{aligned}
& f_{\downarrow}\left(\varrho_{X E}\right)-f_{\downarrow}\left(\sigma_{X E}\right)=F\left(\varrho_{X E}, \mathcal{M}_{\varrho}\right)-F\left(\sigma_{X E}, \mathcal{M}_{\sigma}\right) \leq F\left(\varrho_{X E}, \mathcal{M}_{\sigma}\right)-F\left(\sigma_{X E}, \mathcal{M}_{\sigma}\right)= \\
& =\sum_{k} p_{k} f\left(\varrho_{X}^{k}\right)-\sum_{k} q_{k} f\left(\sigma_{X}^{k}\right) \leq\left|\sum_{k} p_{k} f\left(\varrho_{X}^{k}\right)-\sum_{k} q_{k} f\left(\sigma_{X}^{k}\right)\right|= \\
& =\left|\sum_{k} p_{k} f\left(\varrho_{X}^{k}\right)-p_{k} f\left(\sigma_{X}^{k}\right)+p_{k} f\left(\sigma_{X}^{k}\right)-q_{k} f\left(\sigma_{X}^{k}\right)\right| \leq \\
& \leq \sum_{k}\left(p_{k}\left|f\left(\varrho_{X}^{k}\right)-f\left(\sigma_{X}^{k}\right)\right|+\left|p_{k}-q_{k}\right|\left|f\left(\sigma_{X}^{k}\right)\right|\right) \leq \\
& \leq \sum_{k} p_{k} \varepsilon_{k} K \log d_{X}+\varepsilon M \log d_{X}+O(\varepsilon) \leq K_{1} \varepsilon \log d_{X}+O(\varepsilon)
\end{aligned}
$$

where $\varepsilon_{k}=\left\|\varrho_{X}^{k}-\sigma_{X}^{k}\right\|_{1}$ and $K_{1}=2 K+M$. The last two steps of the above estimate are implied by asymptotic continuity, subextensivity of the function $f$ and the following facts (see [23]):

$$
\sum_{k}\left|p_{k}-q_{k}\right| \leq \varepsilon
$$

and

$$
\sum_{k} p_{k} \varepsilon_{k} \leq 2 \varepsilon
$$

The inequality (43) we get via the following estimate

$$
\begin{aligned}
& \sum_{k}\left|p_{k}-q_{k}\right|=\| \sum_{k} p_{k}|k\rangle\left\langle k\left|-\sum_{k} q_{k}\right| k\right\rangle\left\langle k\left|\left\|_{1} \leq\right\| \sum_{k} p_{k} \varrho_{X}^{k} \otimes\right| k\right\rangle\left\langle k\left|-\sum_{k} q_{k} \sigma_{X}^{k} \otimes\right| k\right\rangle\langle k| \|_{1}= \\
& \left\|\left(I_{X} \otimes \Lambda_{\sigma}\right) \varrho_{X E}-\left(I_{X} \otimes \Lambda_{\sigma}\right) \sigma_{X E}\right\|_{1} \leq\left\|\varrho_{X E}-\sigma_{X E}\right\|_{1}=\varepsilon .
\end{aligned}
$$

where $\Lambda_{\sigma}$ is a completely positive map induced by POVM $\mathcal{M}_{\sigma}$ as follows

$$
\Lambda_{\sigma}(\cdot)=\sum_{k} \operatorname{Tr}\left[A_{k}^{\sigma}(\cdot) A_{k}^{\sigma \dagger}\right]|k\rangle\langle k|
$$

We have used here the fact that trace norm does not increase under completely positive trace preserving maps [36]. The inequality (44) is proven as follows

$$
\begin{aligned}
& \varepsilon=\left\|\varrho_{X E}-\sigma_{X E}\right\|_{1} \geq \sum_{k} \| p_{k} \varrho_{X}^{k} \otimes|k\rangle\left\langle k\left|-q_{k} \sigma_{X}^{k} \otimes\right| k\right\rangle\left\langle k\|\|_{1}=\sum_{k}\left\|p_{k} \varrho_{X}^{k}-q_{k} \sigma_{X}^{k}\right\|_{1}\right. \\
& \geq \sum_{k}\left(\left\|p_{k} \varrho_{X}^{k}-p_{k} \sigma_{X}^{k}\right\|_{1}-\left\|p_{k} \sigma_{X}^{k}-q_{k} \sigma_{X}^{k}\right\|_{1}\right) \\
& \left.=\sum_{k} p_{k}\left\|\varrho_{X}^{k}-\sigma_{X}^{k}\right\|_{1}-\sum_{k}\left|p_{k}-q_{k}\right|\right) \geq \sum_{k} p_{k} \varepsilon_{k}-\varepsilon
\end{aligned}
$$

Analogously we can show that

$$
f_{\downarrow}\left(\sigma_{X E}\right)-f_{\downarrow}\left(\varrho_{X E}\right)=F\left(\sigma_{X E}, \mathcal{M}_{\sigma}\right)-F\left(\varrho_{X E}, \mathcal{M}_{\varrho}\right) \leq F\left(\sigma_{X E}, \mathcal{M}_{\varrho}\right)-F\left(\varrho_{X E}, \mathcal{M}_{\varrho}\right) \leq K_{1} \varepsilon \log d_{X}+O(\varepsilon)
$$

Thus we obtain

$$
\mid f_{\downarrow}\left(\varrho^{X E}\right)-f_{\downarrow}\left(\sigma^{X E} \mid \leq K_{1} \varepsilon \log d_{X}+O(\varepsilon)\right.
$$

This ends the proof.

Remark. In the proof we have used the fact that the infimum in definition of $f_{\downarrow}$ is achievable. However it is not essential: the proof that does not use it is very similar to the above one.

Finally, consider modification of the function $f_{\downarrow}$, where we do not optimize over all POVM's, but only over complete POVM's, for which the operators $A_{k}$ are of rank one. 
Definition 6 For any function $f: \mathcal{S}\left(\mathcal{H}_{X}\right) \rightarrow R$ acting on states of system $X$, we define function $f_{\downarrow}^{c p l}: \mathcal{S}\left(\mathcal{H}_{X} \otimes \mathcal{H}_{E}\right) \rightarrow$ $R$ as follows

$$
f_{\downarrow}^{c p l}\left(\rho_{X E}\right)=\inf _{\left\{A_{i}\right\}} \sum_{i} p_{i} f\left(\rho_{X}^{i}\right)
$$

where infimum is taken over all finite POVM's $\left\{A_{i}\right\}$ with elements $A_{i}$ being of rank one. The notation is the same as in Def. 3

Again, the infimum in the above definition can be achieved, see Sec. [X] We then obtain

Proposition 3 Let $f$ be a function defined on states of system $X$, which is subextensive and asymptotic continuous. Then function $f_{\downarrow}^{\text {cpl }}$ is also asymptotically continuous. Moreover, the constant in asymptotic continuity condition depends only on dimension of system $X$.

The proof is analogous to the proof of Prop. 2

\section{APPLICATIONS}

\section{A. Measure of classical correlation $C_{\leftarrow}$}

This proposition implies asymptotic continuity of measure of classical correlation $C_{\leftarrow}$ defined as follows $[9]$ :

$$
C_{\leftarrow}\left(\rho_{A B}\right)=\max _{B_{i}^{\dagger} B_{i}} S\left(\rho_{A}\right)-\sum_{i} p_{i} S\left(\rho_{A}^{i}\right)
$$

where $B_{i}^{\dagger} B_{i}$ is a POVM performed on subsystem $B, \rho_{A}^{i}=\operatorname{tr}_{B}\left(I \otimes B_{i} \rho_{A B} I \otimes B_{i}^{\dagger}\right) / p_{i}$ is remaining state of $A$ after obtaining the outcome $i$ on $B$, and $p_{i}=\operatorname{tr}_{A B}\left(I \otimes B_{i} \rho_{A B} I \otimes B_{i}^{\dagger}\right)$. Notice that we can rewrite $C_{\leftarrow}$ :

$$
C_{\leftarrow}\left(\varrho_{A B}\right)=\max _{B_{i}^{\dagger} B_{i}} \sum_{i} p_{i}\left(S\left(\sum_{i} p_{i} \varrho_{A}^{i}\right)-S\left(\varrho_{A}^{i}\right)\right)
$$

So $C_{\leftarrow}$ is a kind of function build by "arrowing", where $f: \mathcal{S}\left(\mathcal{H}_{A}\right) \rightarrow R$ acting on states of system $A$ if of the form:

$$
f\left(\varrho_{A}^{i}\right)=S\left(\sum_{i} p_{i} \varrho_{A}^{i}\right)-S\left(\varrho_{A}^{i}\right)
$$

Function $f$ is asymptotically continous, beacuse entropy von Neumann $S$ possess this feature. So whereby of Proposition 2 quantity $C_{\leftarrow}$ is also asymptotically cointinous.

\section{B. Intrinsic conditional information}

Consider the following function called intrinsic conditional information: $I(X ; Y \downarrow E)[26]$ between $\mathrm{X}$ and Y given E defined as

$$
I(X ; Y \downarrow E)=\inf _{P_{\bar{E} \mid E}} I(X ; Y \mid \bar{E})=\inf _{P_{\bar{E} \mid E}} \sum_{e} p(\bar{e}) I(X ; Y \mid \bar{E}=\bar{e})
$$

where $P_{\bar{E} \mid E}$ is a classical channel, $I(X ; Y \mid \bar{E}=\bar{e})$ is mutual information between $\mathrm{X}$ and $\mathrm{Y}$ given $\bar{E}=\bar{e}$ and $p(\bar{e})$ is probability that we have outcome $\bar{e}$ on subsystem $\bar{E}$. The quantity $I(X ; Y \mid \bar{E})=\sum_{e} p(\bar{e}) I(X ; Y \mid \bar{E}=\bar{e})$ is called conditional information. It is known [37] that infimum in definition of intrinsic conditional information is achievable. It is enough to take minimum over $P_{\bar{E} \mid E}$ with the system $\bar{E}$ of size of $E$.

One easily finds, that that intrinsic information is a particular case of "arrowing". Indeed, for a given classical channel $P_{\bar{E} \mid E}$ with conditional probabilities $\left\{p_{\bar{e} \mid e}\right\}$ we consider POVM given by Kraus operator $A_{\bar{e}}=\sum_{e} \sqrt{p_{\bar{e} \mid e}}|e\rangle\langle e|$. Now, if we embedded in natural way our distribution into set of quantum states, then we see that our definition 3 reproduces the above quantity.

If we notice that the mutual information itself is asymptotically continuous ( it is sum of entropies, each of them being asymptotically continuous due to Fannes inequality (10), then we will see that the asymptotic continuity of intrinsic conditional information follows from our theorem. 


\section{CONVEX ROOF FUNCTIONS}

Here we present asymptotic continuity of functions constructed from other asymptotically continuous function $f$ by means of convex roof [27]. We will distinguish between pure and mixed convex roof. The pure convex roof is generalization of definition of entanglement of formation $E_{F}$ given in $[3]$. It was proposed and investigated in Ref. [27] and called there just convex roof.

\section{A. Pure convex roof}

Definition 7 For a function $f$ defined on pure states its pure convex roof $\widehat{f}$ is a function defined on all states, given by

$$
\widehat{f}(\varrho)=\inf _{\left\{p_{k}, \psi_{k}\right\}} \sum_{k} p_{k} f\left(\psi_{k}\right)
$$

where infimum is taken over all finite pure ensembles $\left\{p_{k}, \psi_{k}\right\}$, satisfying $\varrho=\sum p_{k}\left|\psi_{k}\right\rangle\left\langle\psi_{k}\right|$.

It is useful to represent convex roof in a different way (cf. [19]), to make explicit, that operation of pure convex roof is actually arrowing. Indeed, for any state $\varrho$ acting on Hilbert space $\mathcal{H}_{X}$ of dimension $d_{X}$ we can construct its purification i.e. pure state $\varphi_{\varrho}$ acting on Hilbert space $\mathcal{H}_{X} \otimes \mathcal{H}_{E}$ (with $\left.\operatorname{dim} \mathcal{H}_{E}=\operatorname{dim} \mathcal{H}_{X}\right)$ such that

$$
\operatorname{Tr}_{\mathcal{H}_{a n c}} \varphi_{\varrho}=\varrho
$$

Moreover for any pure decomposition of $\varrho$, given by $\left\{p_{k}, \psi_{k}\right\}$ there exists a complete POVM on $\mathcal{H}_{\text {anc }}$ which gives such ensemble on system $X$, and vice versa: any POVM gives rise to some pure decomposition.

Then we can rewrite $\widehat{f}$ as infimum over measurements $\mathcal{M}$

$$
\widehat{f}(\varrho)=\inf _{\sum p_{k}\left|\psi_{k}\right\rangle\left\langle\psi_{k}\right|=\varrho} \sum_{k} p_{k} f\left(\psi_{k}\right)
$$

Consequently, we have

$$
\widehat{f}\left(\varrho_{X}\right)=f_{\downarrow}^{c p l}\left(\varphi_{X E}^{\varrho}\right)
$$

where the equality holds for arbitrarily fixed purification $\varphi_{X E}^{\varrho}$ of the state $\varrho_{X}$. Having rewritten pure convex roof in terms of arrowed function, we can easily prove its asymptotic continuity, by use of the proposition 2

Proposition 4 Let $f$ be a function, which is subextensive and asymptotically continuous. Then its convex roof $\widehat{f}$ is also asymptotically continuous.

Proof.

We will use following inequalities [38]:

$$
1-F(\varrho, \sigma) \leq \frac{1}{2}\|\varrho-\sigma\|_{1} \leq \sqrt{1-F(\varrho, \sigma)}
$$

where $F(\varrho, \sigma)=\sqrt{\sqrt{\varrho} \sigma \sqrt{\varrho}}$ is fidelity [39, 40]. The fidelity can be also expressed as follows

$$
F(\varrho, \sigma)=\sup \left|\left\langle\psi_{\varrho} \mid \psi_{\sigma}\right\rangle\right|
$$

where supremum is taken over all $\psi_{\varrho}$ and $\psi_{\sigma}$ which are purifications of states $\varrho$ and $\sigma$. The supremum is achievable.

Consider now arbitrary states $\varrho$ and $\sigma$ let $\varepsilon=\|\varrho-\sigma\|_{1}$. We want to estimate $\widehat{f}(\varrho)-\widehat{f}(\sigma)$. Since the representation (61) does not depend on the choice of purification, we take such purifications $\psi_{\varrho}$ and $\psi_{\sigma}$, that

$$
F(\varrho, \sigma)=F\left(\psi_{\varrho}, \psi_{\sigma}\right)
$$

Then we have

$$
\|\left|\psi_{\varrho}\right\rangle\left\langle\psi_{\varrho}|-| \psi_{\sigma}\right\rangle\left\langle\psi_{\sigma}\right| \|_{1} \leq 2 \sqrt{1-F\left(\psi_{\varrho}, \psi_{\sigma}\right)}=2 \sqrt{1-F(\varrho, \sigma)} \leq 2 \sqrt{\|\varrho-\sigma\|_{1} / 2}=\sqrt{2 \varepsilon}
$$

Since we assume that $f$ is asymptotically continuous and subextensive, we can use Prop. 2 to get

$$
|\widehat{f}(\varrho)-\widehat{f}(\sigma)|=\left|f_{\downarrow}^{c p l}\left(\psi_{\varrho}\right)-f_{\downarrow}^{c p l}\left(\psi_{\sigma}\right)\right| \leq K \sqrt{2 \varepsilon} \log d_{X}+O(\sqrt{2 \varepsilon})
$$

This ends the proof.

Remark. Notice that however we have here $\sqrt{2 \varepsilon}$ instead of $\varepsilon$, but we think that it does not change essence of condition referring asymptotic continuity. 


\section{MIXED CONVEX ROOF}

Analogously to pure convex roof we can define mixed convex roof.

Definition 8 Let $f$ be a function and $\varrho$ be a state then we can define function mixed convex roof $\widehat{f}$ as follows

$$
\widehat{f}(\varrho)=\inf _{\left\{p_{k}, \varrho_{k}\right\}} \sum_{k} p_{k} f\left(\varrho_{k}\right)
$$

where infimum is taken over all ensembles $\left\{p_{k}, \varrho_{k}\right\}$, where $\varrho=\sum p_{k} \varrho_{k}$.

Similarly as in the case of pure convex roof we can show that

$$
\widehat{f}\left(\varrho_{X}\right)=f_{\downarrow}\left(\psi_{X E}^{\varrho}\right)
$$

where, again, $\psi_{X E}^{\varrho}$ is arbitrarily fixed purification of $\varrho_{X}$.

Therefore, with analogous proof as that of Prop. [4 we obtain

Proposition 5 Let $f$ be subextensive and asymptotically continuous function then function mixed convex roof $\widehat{f}$ is also asymptotically continuous.

\section{APPLICATIONS}

\section{A. Pure convex roof of measure of entanglement for tripartite pure states}

Consider the quantity $E$ [31] which is equal to sum of measure of entanglement for bipartite state applied for subsystem of tripartite state:

$$
E\left(\varrho_{A B C}\right)=E_{R}\left(\varrho_{A B}\right)+S\left(\varrho_{C}\right)
$$

where $\mathrm{S}$ is von Neumann entropy and $\varrho_{A B}=\operatorname{Tr}_{C} \varrho_{A B C}, \varrho_{C}=\operatorname{Tr}_{A B} \varrho_{A B C}$ and $E_{R}$ is relative entropy distance from set of separable states. Now, we can consider pure convex roof of function $E$ :

$$
\widehat{E}\left(\varrho_{A B C}\right)=\inf _{\varrho_{A B C}=\Sigma p_{k}\left|\psi^{k}\right\rangle\left\langle\left.\psi^{k}\right|_{A B C}\right.} \sum_{k} p_{k} E\left(\left|\psi_{A B C}^{k}\right\rangle\right)
$$

Note that $E$ is subextensive and asymptotically continuous, because relative entropy distance and entropy possess these feature. Thus the Proposition 4 implies that convex roof of this function $\widehat{E}$ is also asymptotically continuous.

\section{B. Entanglement of formation}

Proposition 4 implies asymptotic continuity of entanglement of formation $E_{F}$ (which was first shown in [41]) defined as $[\underline{3}]$

$$
E_{F}\left(\varrho_{A B}\right)=\inf _{\varrho_{A B}=\Sigma p_{k}\left|\psi_{k}\right\rangle\left\langle\psi_{k}\right|} \sum_{k} p_{k} S_{A}\left(\left|\psi_{k}\right\rangle\right)
$$

where $S_{A}$ is a von Neumann entropy of subsystem A of state. In original definition infimum is taken over all pure ensembles, but notice that in this case infimum over all ensembles reduce to infimum over pure ensembles. Thus

$$
E_{F}\left(\varrho_{A B}\right)=\inf _{\varrho_{A B}=\Sigma p_{k} \varrho_{k}} \sum_{k} p_{k} S_{A}\left(\varrho_{k}\right)
$$

This is implied by concavity of von Neumann entropy:

$$
\sum_{k} p_{k} S_{A}\left(\varrho_{k}\right)=\sum_{k} p_{A} S_{A}\left(\sum_{i} q_{i}^{k}\left|\varphi_{i}^{k}\right\rangle\left\langle\varphi_{i}^{k}\right|\right) \geq \sum_{k} p_{k} \sum_{i} q_{i}^{k} S_{A}\left(\left|\varphi_{i}^{k}\right\rangle\right)=\sum_{k, i} p_{k} q_{i}^{k} S_{A}\left(\left|\varphi_{i}^{k}\right\rangle\right)
$$

So for every mixed ensemble we can find a pure ensemble which gives no grater value of function $E_{F}$ than a mixed ensemble. 


\section{Pure and mixed convex roof of mutual information.}

Now, we show example of function for which pure and mixed convex roof are not equal to each other. Consider the following functions:

$$
\begin{aligned}
& \widehat{I_{M}}\left(\varrho_{A B}\right)=\inf _{\varrho_{A B}=\Sigma p_{k}\left|\psi_{k}\right\rangle\left\langle\psi_{k}\right|} \sum_{k} p_{k} I_{M}\left(\left|\psi_{k}\right\rangle\right) \\
& \widehat{I_{M}}\left(\varrho_{A B}\right)=\inf _{\varrho_{A B}=\Sigma p_{k} \varrho_{k}} \sum_{k} p_{k} I_{M}\left(\varrho_{k}\right)
\end{aligned}
$$

where $I_{M}$ is mutual information $I_{M}=S_{A}\left(\varrho_{A B}\right)+S_{B}\left(\varrho_{A B}\right)-S\left(\varrho_{A B}\right)$. In our terminology, the functions are pure and convex roof of quantum mutual information. The second one was introduced in [25]. Notice that for a pure convex roof we have

$$
\widehat{I_{M}}\left(\varrho_{A B}\right)=2 \inf _{\varrho_{A B}=\Sigma p_{k}\left|\psi_{k}\right\rangle\left\langle\psi_{k}\right|} \sum_{k} p_{k} S_{A}\left(\left|\psi_{k}\right\rangle\right)=2 E_{F}\left(\varrho_{A B}\right)
$$

Let $\varrho_{a s}$ be antysymmetric state state:

$$
\varrho_{a s}=\frac{1}{d^{2}-d}(I-V)
$$

where $\mathrm{V}$ is a unitary flip operator $\mathrm{V}$ acting on Hilbert space $\mathcal{C}^{d} \otimes \mathcal{C}^{d}$ system defined by $V \phi \otimes \varphi=\varphi \otimes \phi$. We know that [42]

$$
E_{F}\left(\varrho_{a s}\right)=1
$$

So $\widehat{I_{M}}\left(\varrho_{a s}\right)=2$. Then we have the following inequality

$$
\widetilde{I_{M}}\left(\varrho_{a s}\right) \leq I_{M}\left(\varrho_{a s}\right)=2 \log d-S\left(\varrho_{a s}\right)=\log \frac{2 d}{d-1}
$$

So for $d \geq 3$ we have that $\widehat{I_{M}}\left(\varrho_{a s}\right) \neq \widehat{I_{M}}\left(\varrho_{a s}\right)$.

\section{ACHIEVING INFIMUM IN DEFINITION OF ARROWING}

We prove that in definition of arrowing the infimum is achievable, so that it can be replaced by minimum. First we prove the following lemma.

Lemma 4 Let $\left\{p_{i}\right\}$ be a probability distribution then any convex combination $\sum_{i} p_{i} x_{i}$, where $x_{i}=\left(\varrho_{i}, f\left(\varrho_{i}\right)\right)$, equal to $\sum p_{i}\left(\varrho_{i}, f\left(\varrho_{i}\right)\right)$ can be written as a convex combination $\sum q_{i}\left(\varrho_{i}, f\left(\varrho_{i}\right)\right)$ consisting of $n+1$ (or less) ingredients, where $n$ is a dimension of space on which is acting $x_{i}$. So

$$
\sum_{i} p_{i} \varrho_{i}=\sum_{i=1}^{n+1} q_{i} \varrho_{i} \quad \text { and } \quad \sum_{i} p_{i} f\left(\varrho_{i}\right)=\sum_{i=1}^{n+1} q_{i} f\left(\varrho_{i}\right)
$$

Proof. Let $\tilde{f}=\sum_{i} p_{i} f\left(\varrho_{i}\right)$ where $\varrho=\sum_{i} p_{i} \varrho_{i}$ is a state acting on Hilbert space $\mathcal{H}$. Let $x_{i}=\left(\varrho_{i}, f\left(\varrho_{i}\right)\right)$ be a point from a convex set $\mathcal{S}=\operatorname{co}\left(\varrho_{i}, f\left(\varrho_{i}\right)\right)$. Then

$$
(\varrho, \tilde{f})=\left(\sum_{i} p_{i} \varrho_{i}, \sum_{i} p_{i} f\left(\varrho_{i}\right)\right)=\sum_{i} p_{i}\left(\varrho_{i}, f\left(\varrho_{i}\right)\right) \in \mathcal{S}
$$

Using Caratheodory 's Theorem we have that there exists such set of probability distribution consisting of $n+1$ or less elements that

$$
(\varrho, \tilde{f})=\sum_{i} q_{i}\left(\varrho_{i}, f\left(\varrho_{i}\right)\right)
$$


So $\varrho=\sum_{i} q_{i} \varrho_{i}$ and $\tilde{f}=\sum_{i} q_{i} f\left(\varrho_{i}\right)$. This ends the proof.

Now, we use above lemma to proof that infimum in function $f_{\downarrow}\left(\varrho_{X E}\right)$ is achievable. Let $\psi_{A X E}$ be a purification of state $\varrho_{X E}$. Then if we make measurement $\mathcal{M}$ on subsystem $E$ we get ensemble $\left\{p_{i}, \varrho_{i}^{A X}\right\}$ on subsystem $A X$. Lets define function $\tilde{f}$ such that for any given function $f$

$$
\tilde{f}\left(\varrho_{i}^{A X}\right)=f\left(\varrho_{i}^{X}\right)
$$

where $\varrho_{i}^{X}=\operatorname{Tr}_{A} \varrho_{i}^{A X}$ Then

$$
f_{\downarrow}\left(\varrho^{X E}\right)=\inf _{\mathcal{M}} \sum_{i} p_{i} f\left(\varrho_{i}^{X}\right)=\inf _{\mathcal{M}} \sum_{i} p_{i} \tilde{f}\left(\varrho_{i}^{A X}\right)
$$

Notice that for function $\tilde{f}$ and state $\psi_{A X E}$ we can define

$$
f_{\downarrow}\left(\psi_{A X E}\right)=\inf _{\mathcal{M}} \sum_{i} p_{i} \tilde{f}\left(\varrho_{i}^{A X}\right)
$$

where we treat subsystem $A X$ as a one subsystem and $E$ as a second. Note also that

$$
f_{\downarrow}\left(\psi_{A X E}\right)=\inf _{\left\{p_{i}, \varrho_{i}^{X}{ }_{E}\right\}} \sum_{i} p_{i} \tilde{f}\left(\varrho_{i}^{A E}\right)
$$

because we can always find such measurement made on subsystem $E$ of state $\psi_{A X E}$, which give us ensemble $\left\{q_{i}, \varrho_{i}^{A X}\right\}$.

Then using Lemma 4 we know that there exists other finite ensemble $\left\{q_{i}, \varrho_{i}^{A X}\right\}$ such that

$$
\sum_{i} p_{i} \varrho_{i}^{A X}=\sum_{i}^{d+1} q_{i} \varrho_{i}^{A X} \quad \text { and } \quad \sum_{i} p_{i} \tilde{f}\left(\varrho_{i}^{A X}\right)=\sum_{i}^{d+1} q_{i} \tilde{f}\left(\varrho_{i}^{A X}\right)
$$

where $d$ is dimension of space on which is acting $\sum_{i} p_{i} \varrho_{i}^{A X}$. So for function $f_{\downarrow}\left(\psi_{A X E}\right)$ infimum over measurement is effectively equal to infimum over bounded finite set of ensembles, so we have infimum over compact states. This implies that there exists extremal point belonging to $\mathcal{S}$, so infimum for this function is achievable. If we are loking at formules (84) and (85) we can see that $f_{\downarrow}\left(\psi_{A X E}\right)=f_{\downarrow}\left(\varrho^{X E}\right)$, what implies that for any given state $\varrho^{X E}$ function $f_{\downarrow}\left(\varrho^{X E}\right)$ achieves infimum.

Acknowledgments. We would like to thank Andreas Winter for helpful discussion on achievability of infima. This work is supported by Polish Ministry of Scientic Research and Information Technology under the (solicited) grant no. PBZ-MIN-008/P03/2003, EU grants RESQ (IST-2001-37559), QUPRODIS (IST-2001-38877) and EC IP SCALA.

\section{APPENDIX}

Now we will present other version of Proposition 1 We will use Cauchy type conditions for asymptotic continuity and show that they are also equivalent to robustness under admixtures.

Proposition 6 Let $f$ be a function, then the following conditions are equivalent:

$$
\begin{array}{lllll}
\text { 1) } \forall_{\varepsilon>0} & \exists_{\delta>0} & \forall_{\varrho, \sigma} & \|\varrho-\sigma\|_{1} \leq \delta \Longrightarrow|f(\varrho)-f(\sigma)| \leq K_{1} \varepsilon \log d+O(\varepsilon) \\
\text { 2) } \forall_{\varepsilon>0} & \exists_{\delta>0} & \forall_{\varrho, \sigma} & |f((1-\delta) \varrho+\delta \sigma)-f(\varrho)| \leq K_{2} \varepsilon \log d+O(\varepsilon)
\end{array}
$$

$K_{1}, K_{2}$ are constants and $O(x)$ is any function that satisfies (i) $O(x)$ converges to 0 when $x$ converges to 0 and (i) $O(x)$ depends only on $x$ (so in our particular case, it will not depend on dimension).

Proof.

$" 1 \Rightarrow 2$ " Let $\varepsilon>0$ be fix then there exists such $\delta>0$ that for any states $\varrho$ and $\sigma$, the following conditions is fulfilled

$$
\|\varrho-\sigma\|_{1} \leq \delta \Longrightarrow|f(\varrho)-f(\sigma)| \leq K_{1} \varepsilon \log d+O(\varepsilon)
$$

Notice that there exists such $\delta_{1}=\frac{\delta}{2}$

$$
\left\|\varrho-\left(\left(1-\delta_{1}\right) \varrho+\delta_{1} \sigma\right)\right\|_{1}=\delta_{1}\|\varrho-\sigma\|_{1} \leq 2 \delta_{1}=\delta
$$


this implies that

$$
\left|f\left(\left(1-\delta_{1}\right) \varrho+\delta_{1} \sigma\right)-f(\varrho)\right| \leq K_{1} \varepsilon \log d+O(\varepsilon)=K_{2} \varepsilon \log d+O(\varepsilon)
$$

$" 2 \Rightarrow 1 "$

Let $\varepsilon>0$ then there exists such $\delta>0$ that

$$
\forall_{\varrho, \sigma} \quad|f((1-\delta) \varrho+\delta \sigma)-f(\varrho)| \leq K_{2} \varepsilon \log d+O(\varepsilon)
$$

Let $\varrho_{1}, \varrho_{2}$ be state that

$$
\left\|\varrho_{1}-\varrho_{2}\right\|_{1}=\delta_{1} \leq \delta
$$

Analogously to proof of Theorem 1

$$
\begin{gathered}
\exists_{\sigma, \gamma_{1} \gamma_{2}} \quad \sigma=\left(1-\delta_{1}\right) \varrho_{1}+\delta_{1} \gamma_{1}=\left(1-\delta_{1}\right) \varrho_{2}+\delta_{1} \gamma_{2} \\
\left|f\left(\varrho_{2}\right)-f\left(\varrho_{1}\right)\right| \leq\left|f\left(\varrho_{2}\right)-f(\sigma)\right|+\left|f(\sigma)-f\left(\varrho_{1}\right)\right|= \\
\left|f\left(\varrho_{2}\right)-f\left(\left(1-\delta_{1}\right) \varrho_{2}+\delta_{1} \gamma_{2}\right)\right|+\left|f\left(\left(1-\delta_{1}\right) \varrho_{1}+\delta_{1} \gamma_{1}\right)-f\left(\varrho_{1}\right)\right| \leq 2 K_{2} \log d+2 O(\varepsilon)=K_{1} \log d+O(\varepsilon)
\end{gathered}
$$

This ends the proof.

[1] C. H. Bennett, D. P. DiVincenzo, and J. A. Smolin, Phys. Rev. Lett 78, 3217 (1997), quant-ph/9701015.

[2] C. H. Bennett, I. Devetak, P. W. Shor, and J. A. Smolin, quant-ph/0406086.

[3] C. H. Bennett, D. P. DiVincenzo, J. Smolin, and W. K. Wootters, Phys. Rev. A 54, 3824 (1997), quant-ph/9604024.

[4] P. Hayden, M. Horodecki, and B. Terhal, J. Phys. A 34, 6891 (2001), quant-ph/0008134.

[5] B. Schumacher, Phys. Rev. A 51, 2738 (1995).

[6] J. Oppenheim, M. Horodecki, P. Horodecki, and R. Horodecki, Phys. Rev. Lett 89, 180402 (2002), quant-ph/0112074.

[7] M. Horodecki, P. Horodecki, R. Horodecki, J. Oppenheim, A. Sen(De), U. Sen, and B. Synak, Phys. Rev. A 71, 062307 (2005), quant-ph/0410090.

[8] G. Vidal, J. Mod. Opt. 47, 355 (2000), quant-ph/9807077.

[9] L. Henderson and V. Vedral, J. Phys. A 34, 6899 (2001), quant-ph/0105028.

[10] B. M. Terhal, M. Horodecki, D. P. DiVincenzo, and D. Leung, J. Math. Phys. 43, 4286 (2002), quant-ph/0202044.

[11] I. Devetak and A. Winter, quant-ph/0304196.

[12] B. Synak and M. Horodecki, J. Phys. A 37, 11465 (2004), quant-ph/0403167.

[13] M. Fannes, Commun. Math. Phys. 31, 291 (1973).

[14] M. Horodecki, Phys. Rev. A 57, 3364 (1998), quant-ph/9712035.

[15] H. Barnum, C. M. Caves, C. A. Fuchs, R. Jozsa, and B. W. Schumacher, quant-ph/0008024.

[16] M. Horodecki, P. Horodecki, and R. Horodecki, Phys. Rev. Lett 84, 2014 (2000), quant-ph/9908065.

[17] M. Donald, M. Horodecki, and O. Rudolph, J. Math. Phys. 43, 4252 (2002), quant-ph/0105017.

[18] M. Horodecki, Quantum Inf. Comp. 1, 3 (2001).

[19] M. A. Nielsen, Phys. Rev. A 61, 064301 (2000), quant-ph/9908086.

[20] M. Donald and M. Horodecki, Phys. Lett. A 264, 257 (2001), quant-ph/9910002.

[21] V. Vedral and M. B. Plenio, Phys. Rev. A 57, 1619 (1998), quant-ph/9707035.

[22] R. Alicki and M. Fannes, J. Phys. A 37 (2003), quant-ph/0312081.

[23] M. Christandl and A. Winter, quant-ph/0308088.

[24] K. Horodecki, M. Horodecki, P. Horodecki, and J. Oppenheim, quant-ph/0404096.

[25] M. Christandl, Diploma Thesis, Institute for Theoretical Computer Science,ETH Zurich (2002).

[26] U. Maurer and S. Wolf, IEEE Transactions on Information Theory 45, 499514 (1999).

[27] A. Uhlmann, Open Sys. Inf. Dyn. 5, 209 (1998).

[28] D. Yang, M. Horodecki, R. Horodecki, and B. Synak-Radtke, quant-ph/0506138.

[29] N. Linden, S. Popescu, B. Schumacher, and M. Westmoreland (1999), quant-ph/9912039.

[30] H.Araki and H.Moriya (2003), unpublished.

[31] N. Linden, S. Popescu, B. Schumacher, and M. Westmoreland, quant-ph/9912039.

[32] A.Wehrl, Reviews of Modern Physics 50, 221 (1978).

[33] U. Maurer and S. Wolf, Lecture Notes in Computer Science 1807, 351 (2000).

[34] M. Christandl and R. Renner, in Proceedings of 2004 IEEE International Symposium on Information Theory (IEEE, 2004), p. 135.

[35] T.Moroder, M.Curty, and N.Lutkenhaus, quant-ph/0507235. 
[36] M. B. Ruskai, Rev. Math. Phys. 6, 1147 (1994).

[37] M. Christandl, R. Renner, and S. Wolf (2002), www.citeseer.ist.psu.edu/christandl03property.html.

[38] C. A. Fuchs and J. van de Graaf, IEEE Transactions on Information Theory 45, 1216 (1999), quant-ph/9712042.

[39] A. Uhlmann, Rep. Math. Phys. 9, 273 (1976).

[40] R. Jozsa, J. Mod. Opt. 41, 2315 (1994).

[41] M. A. Nielsen, Physical Review A 61, 064301 (2000), quant-ph/9908086.

[42] K.Vollbrecht and R.F.Werner, Phys. Rev. A 64, 0623074 (2001), quant-ph/0010095. 